\title{
¿Se deberían replantear las recomendaciones relativas al uso sedentario del tiempo de pantalla en adolescentes?
}

\section{Should we reconsider sedentary screen time recommendations in adolescents?}

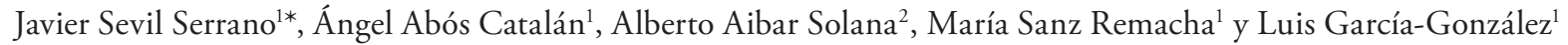 \\ 1 Facultad de Ciencias de la Salud y del Deporte, Universidad de Zaragoza, (España). \\ 2 Facultad de Ciencias Humanas y de la Educación, Universidad de Zaragoza (España).
}

\begin{abstract}
Resumen: El objetivo fue analizar el tiempo medio de uso diario de cuatro medios tecnológicos de pantalla (i.e., TV, ordenador, videojuegos y móvil) e identificar el grado de cumplimiento de las recomendaciones de pantalla, tanto de manera independiente como en su conjunto. Asimismo, se analizaron las diferencias en función del tipo de jornada y el género. Participaron 105 estudiantes $(M=13.05 \pm 0.59)$ que cumplimentaron un cuestionario referente al tiempo de pantalla de dichos medios tecnológicos. Los adolescentes de esta muestra pasan un tiempo medio de uso diario de 407 minutos de medios tecnológicos de pantalla, siendo la TV el medio más utilizado. Ninguno de los adolescentes cumple las recomendaciones de tiempo de panta1la. Asimismo, existe un mayor de tiempo de uso tecnológico los fines de semana y por parte de los chicos. Se discute la necesidad de actualización de las recomendaciones relativas al uso del tiempo de pantalla en adolescentes. Palabras clave: Tiempo de pantalla, recomendaciones, teléfono móvil, adolescentes.
\end{abstract}

Abstract: The aim of thisstudy was to analyze the daily sedentary screen time of four electronicdevices behaviors (i.e., TV, computer, videogames, and mobile), and the proportion of adolescents who meet recommendations for screen time either independently or combined. Differences according to the type of day and gender were analyzed. One hundred fivestudents $(M=13.05 \pm 0.59)$ completed a self-reported questionnaire about these sedentary screen time behaviors. Adolescents reported an average daily time of 407 minutes of sedentary screen time behaviors, with TV being the most used electronic device. Results showed that all adolescents failed to meet screen time recommendations. Greater values of sedentary screen time behaviors were reported on weekends days and in boys than in weekdays and in girls respectively. The need to update the sedentary screen time guidelines in adolescents is discussed.

Keywords: Screen time, recommendations, mobile phone, adolescents.

\section{Introducción}

Desde hace más de una década prevalecen las recomendaciones establecidas por la American Association of Pediatrics (2001) que establecen que el tiempo de pantalla diario (e.g., televisión [TV], ordenador, videojuegos, etc.) no debe sobrepasar las dos horas en niños y adolescentes. Sin embargo, la emergente aparición y modernización de nuevos dispositivos electrónicos (e.g., smartphones o tablets) en países desarrollados, con permanente conexión a internet, han elevado considerablemente el tiempo que permanecen los adolescentes delante de estas pantallas (Pate, Mitchell, Byun, \& Dowda, 2011; Twenge, Krizan, \& Hisler, 2017).Por ejemplo, actualmente los smartphones son utilizados como despertador, cámara de fotos, videoconsola, teléfono, agenda, chat, entre otras muchas funciones (LeBlanc et al., 2017). Este incesante desarrollo tecnológico es especialmente preocupante en la infancia y adolescencia, ya que una exposición prolongada a algunos de estos dispositivos electrónicos se ha visto asociada a consecuencias negativas en la salud física(e.g., composición corporal desfavorable, riesgo metabólico, mala condición fí-

Dirección para correspondencia [Correspodence address]: Javier Sevil Serrano: Facultad de Ciencias de la Salud y del Deporte, Universidad de Zaragoza, España. Plaza Universidad, 3, 22001, Huesca (España). E-mail: jsevils@unizar.es sica; Carson et al., 2016; Costigan, Barnett, Plotnikoff, \& Lubans, 2013; Lissak, 2018; Tremblay et al., 2011) y psicológica (e.g., menor bienestar, baja autoestima, trastornos de ansiedad y depresión; Carson et al., 2016; Costigan et al., 2013).

Concretamente, la transición entre la etapa de Educación Primaria y Educación Secundaria se ha visto asociada a un incremento tanto de los niveles de comportamiento sedentario como de tiempo de pantalla (Arundell, Fletcher, Salmon, Veitch, \& Hinkley, 2016; Mielgo-Ayuso et al., 2017;Pearson, Haycraft, Johnston, \& Atkin, 2017). Una revisión sistemática previa realizada en diferentes países europeos señaló que el tiempo dedicado a comportamientos sedentarios en los adolescentes se situaba entre 268 y 508 minutos (Verloigne et al., 2016).Sin embargo, estos estudios incluían tanto mediciones del comportamiento sedentario, a través de acelerometría, como cuestionarios auto-reportados en los que se incluía la TV, el ordenador y los videojuegos. En la actualidad, la $S e$ dentary Behavior Research Network ha redactado un informe (Tremblay et al., 2017) en el que diferencian los conceptos de comportamiento sedentario y tiempo de pantalla), estableciendo distintas categorías a su vez para diferenciar el tiempo de pantalla (i.e., tiempo de pantalla recreativo, tiempo de pantalla estacionario, uso sedentario del tiempo de pantalla y uso activo del tiempo de pantalla;). Por ejemplo, no es lo 
mismo medir el tiempo diario de videojuegos activos, que se han visto asociado con el incremento de los niveles de AFMV (Norris, Hamer, \& Stamatakis, 2016) que el tiempo diario de videojuegos pasivos que se asocia negativamente con el desarrollo de conductas prosociales (Carson et al., 2016).

En España, existe una gran disparidad de resultados en relación a la utilización de medios tecnológicos de pantalla en población adolescente, debido probablemente al número y la diferente tipología de aparatos electrónicos utilizados. Valencia-Peris, Devís-Devís, y Peiró-Velert (2014) señalaron que los adolescentes pasaban tres horas diarias utilizando medios tecnológicos de pantalla (i.e., TV, ordenador y videojuegos). Sin embargo, en el estudio de Aibar, Bois, Zaragoza, Bru, y Generelo (2015), en el que se midió el tiempo dedicado ala TV, el ordenador, los videojuegos y el teléfono móvil, se registraron unas cuatro horas diarias de tiempo de pantalla. En sintonía con las diferencias encontradas en el uso diario de tiempo de pantalla, el cumplimiento de las recomendaciones de tiempo de pantalla también muestra una gran disparidad de resultados. En los últimos años numerosos estudios han evaluado el cumplimiento del tiempo de pantalla atendiendo a cada uno de los comportamientos tecnológicos examinados por separado (e.g., Mielgo-Ayuso et at., 2017) y/o mediante la suma de todos ellos (e.g., Valencia et al., 2014). El estudio internacional Health Behavior in School-aged Children (HBSC) realizado en 42 países de Europa y América del Norte mostró que aproximadamente el 63\% de los adolescentes excedía las recomendaciones de tiempo de pantalla (Inchleyet al., 2016). En España, la Encuesta Nacional de Salud de 2011/2012,señaló que el $52.3 \%$ de los niños y adolescentes de 5 a 14 años pasaba entre semana más de 2 horas al día de ocio sedentario de pantalla, siendo esta cifra superior en el género masculino y durante el fin de semana. Analizando concretamente las recomendaciones sobre TV y tiempo de juego (i.e., videojuegos, ordenador u otros dispositivos) en función del tipo de jornada, se aprecia que el $13.1 \%$ y el $4.4 \%$ respectivamente no cumple las recomendaciones entre semana, mientras que el $38.3 \%$ y el $16 \%$ respectivamente no las cumple los fines de semana. Sin embargo, en el estudio realizado por Valencia et al. (2014) el porcentaje de adolescentes que no cumple dichas recomendaciones de tiempo de pantalla se incrementa hasta el $72.8 \%$.

Atendiendo a los cambios vertiginosos que se han producido en los últimos años con relación al tiempo de pantalla, y a la falta de unanimidad en la literatura para calcular las recomendaciones de uso sedentario de tiempo de pantalla, parece necesario seguir investigando en la prevalencia de estos hábitos en población adolescente (Tremblay et al., 2011).En base a ello, el primer objetivo del estudio fue analizar el tiempo diario de uso sedentario de cuatro medios tecnológicos de pantalla (i.e., TV, ordenador, videojuegos y teléfono móvil), e identificar el grado de cumplimiento de las recomendaciones de pantalla, tanto de manera independiente para cada medio como en su conjunto. Asimismo, el segundo objetivo fue analizar diferencias en función del tipo de jornada y el género en el uso de dichos medios tecnológicos de pantalla.

\section{Método}

\section{Diseño y participantes}

Se realizó un diseño transversal descriptivo en el que participaron 105 estudiantes $(M=13.05 ; D T=0.59), 50$ chicos y 55 chicas, de un centro de enseñanza público de la ciudad de Huesca (Aragón, España). Los padres y el propio alumnado firmaron el consentimiento informado antes de comenzar el estudio. El Comité de Ética de la Investigación de la Comunidad de Aragón (CEICA) aprobó la realización del estudio.

\section{Instrumentos}

Se administró un cuestionario auto-reportado (Rey-López et al., 2011) para conocer el tiempo de uso sedentario de los medios tecnológicos de TV, ordenador, videojuegos y teléfono móvil. Para ello, se preguntó por el tiempo diario entre semana y el fin de semana de cada uno de dichos tipos de pantalla. El tiempo medio diario de cada medio tecnológico se calculó haciendo la media entre el tiempo diario entre semana y el fin de semana. Por último, para evaluar los minutos diarios de tiempo de pantalla se sumó la media diaria de los cuatro comportamientos sedentarios.

\section{Análisis de datos}

En primer lugar, se calcularon los estadísticos descriptivos (media, desviación típica y porcentajes) de los cuatro medios tecnológicos de pantalla (i.e., TV, ordenador, videojuegos y teléfono móvil). El grado de cumplimiento del tiempo de pantalla fue calculado, tanto de manera independiente en cada uno de los medios tecnológicos de pantalla, como en su conjunto, utilizando las recomendaciones internacionales para adolescentes ( $<2$ horas de tiempo de pantalla; American Association of Pediatrics, 2001; Tremblay et al., 2016). Se analizaron diferencias en función del género a través de sucesivos análisis de varianza univariados (ANOVAs) en el tiempo diario de los cuatro medios tecnológicos de pantalla. Las diferencias en función del tipo de jornada (semana y fin de semana) se analizaron mediante un modelo lineal general de medidas repetidas. El grado de asociación entre el cumplimiento de tiempo de pantalla de cada uno de los cuatro medios tecnológicos de pantalla y el género se calculó utilizando la prueba Chi-cuadrado $\left(x^{2}\right)$.Concretamente, el coeficiente $\mathrm{V}$ de Cramer se utilizó para estimar el tamaño del efecto, pu- 
diendo ser, de acuerdo a los criterios de Cohen (1988), bajo (.10), medio (.30) o elevado (.50). Por último, para el cálculo del cumplimiento de las recomendaciones de tiempo en pantalla en función del tipo de jornada se utilizó la prueba de McNemar. Todos los análisis se realizaron con el programa estadístico SPSS 21.0.

\section{Resultados}

En la tabla 1, se puede apreciar que los adolescentes de esta muestra pasan una media de 407 minutos diarios de tiempo de pantalla (6 horas y 47 minutos), siendo la TV el medio tecnológico más utilizado, seguido del teléfono móvil, los vi- deojuegos y el ordenador. La utilización de estos dispositivos se mantiene en la misma línea entre semana y el fin de semana, salvo el uso del ordenador que muestra cifras más elevadas que el uso de los videojuegos entre semana. Con relación al tipo de jornada, se aprecian valores significativamente superiores en el tiempo medio diario total y en los cuatro medios tecnológicos de pantalla (i.e., TV, ordenador, videojuegos y teléfono móvil) los fines de semana. Con relación al género, los chicos presentan valores significativamente superiores en el tiempo medio diario total, entre semana y fin de semana y en el tiempo medio diario de videojuegos, entre semana y fin de semana. Sin embargo, no existen diferencias significativas de género en la TV, el ordenador y el teléfono móvil.

Tabla 1. Estadísticos descriptivos del tiempo diario de TV, ordenador, videojuegos y teléfono móvil y análisis de diferencias en función del tipo de jornada y género.

\begin{tabular}{|c|c|c|c|c|}
\hline Variables de estudio $(\mathrm{M} \pm \mathrm{DT})$ & Total $(n=105)$ & Chicos $(n=50)$ & Chicas $(n=55)$ & $\mathrm{F}-\eta_{\mathrm{p}}{ }^{2}$ \\
\hline Minutos diarios de TV & $130.3 \pm 69.3$ & $136.9 \pm 67.0$ & $124.4 \pm 71.4$ & $.855 / .008$ \\
\hline Minutos diarios de TV entre semana & $117.8 \pm 69.3$ & $125.0 \pm 65.6$ & $111.3 \pm 72.6$ & $1.011 / .010$ \\
\hline Minutos diarios de TV el fin de semana & $161.6 \pm 70.9^{* *}$ & $166.80 \pm 72.4$ & $157.0 \pm 69.8$ & $.498 / .005$ \\
\hline Minutos diarios de videojuegos & $83.9 \pm 84.7$ & $122.1 \pm 91.8$ & $49.15 \pm 59.9$ & $23.640^{* *} / .187$ \\
\hline Minutos diarios de videojuegos entre semana & $70.0 \pm 77.9$ & $104.4 \pm 84.9$ & $38.8 \pm 55.4$ & $22.348^{* *} / .178$ \\
\hline Minutos diarios de videojuegos el fin de semana & $118.5 \pm 104.5^{* *}$ & $166.5 \pm 111.7$ & $75.0 \pm 75.0$ & $24.644^{* *} / .193$ \\
\hline Minutos diarios de ordenador & $82.5 \pm 76.0$ & $82.1 \pm 75.9$ & $83.0 \pm 76.8$ & $.004 / .000$ \\
\hline Minutos diarios de ordenador entre semana & $77.9 \pm 76.4$ & $77.9 \pm 75.7$ & $77.9 \pm 77.8$ & $.000 / .000$ \\
\hline Minutos diarios de ordenador el fin de semana & $94.3 \pm 76.1^{* *}$ & $92.7 \pm 77.5$ & $95.8 \pm 75.6$ & $.043 / .000$ \\
\hline Minutos diarios de teléfono móvil $(\mathrm{M} \pm \mathrm{DT})$ & $111.0 \pm 71.14$ & $111.0 \pm 67.9$ & $110.9 \pm 74.5$ & $.000 / .000$ \\
\hline Minutos diarios de teléfono móvil entre semana & $99.2 \pm 71.74$ & $98.70 \pm 65.0$ & $99.8 \pm 77.8$ & $.006 / .000$ \\
\hline Minutos diarios de teléfono móvil el fin de semana & $140.2 \pm 76.4^{* *}$ & $141.8 \pm 82.3$ & $138.9 \pm 71.5$ & $.037 / .000$ \\
\hline Minutos totales diarios de tiempo de pantalla & $407.8 \pm 186.6$ & $452.2 \pm 204.1$ & $367.5 \pm 160.6$ & $5.628 * / .052$ \\
\hline Minutos totales diarios de tiempo de pantalla entre semana & $365.1 \pm 180.2$ & $406.0 \pm 194.5$ & $327.9 \pm 159.0$ & $5.108 * / .047$ \\
\hline Minutos totales diarios de tiempo de pantalla el fin de semana & $514.8 \pm 207.7^{* *}$ & $567.8 \pm 233.4$ & $466.7 \pm 169.3$ & $6.531^{*} / .060$ \\
\hline
\end{tabular}

Nota: ${ }^{*}=p<0.05 ;{ }^{* *}=p<0.01$. Las diferencias en función del género (hombre, mujer) aparecen reflejadas en la parte derecha de la tabla (F- $\left.{ }_{\mathrm{p}}{ }^{2}\right)$. Las diferencias en función del tipo de jornada (semana y fin de semana) se analizaron en la columna de muestra total $(n=105)$ en cada uno de los medios tecnológicos de pantalla (TV, ordenador, videojuegos, móvil y totales). La significatividad se marca en el tipo de jornada de mayor valor.

Por otro lado, en la tabla 2 se aprecia que ninguno de los adolescentes de la muestra cumple diariamente y el fin de semana las recomendaciones de tiempo de pantalla total (se incluye en dichas recomendaciones la suma de los diferentes medios tecnológicos). Solo un 3\% de la muestra cumple las recomendaciones de tiempo de pantalla entre semana. Las recomendaciones menos cumplidas son las de TV, seguidas del teléfono móvil, los videojuegos y el ordenador, oscilando entre valores del $-30 \%$ y el $-80 \%$. El cumplimiento de las recomendaciones de estos dispositivos se mantiene en la misma línea entre semana y el fin de semana. Con relación al tipo de jornada, se aprecia que el cumplimiento de las recomendaciones es significativamente superior en los cuatro medios tecnológicos de pantalla entre semana. Con relación al género, se puede apreciar una asociación positiva entre el género femenino y el cumplimiento de las recomendaciones de videojuegos. Sin embargo, no existe una asociación significativa entre el género y la TV, el ordenador y el teléfono móvil respectivamente. 
Tabla 2. Cumplimiento de las recomendaciones de tiempo de pantalla en la TV, el ordenador, los videojuegos y el teléfono móvil y grado de asociación en función del tipo de jornada y género.

\begin{tabular}{|c|c|c|c|c|}
\hline Variables de estudio( $\% \leq 2$ horas por día) & Total $(n=105)$ & Chicos $(n=50)$ & Chicas $(n=55)$ & $x 2(g l) /$ V Cramer \\
\hline Cumplimiento de las recomendaciones de TV & $39(37.1 \%)$ & $16(32.0 \%)$ & $23(41.8 \%)$ & $1.081(1) / .101$ \\
\hline Cumplimiento de las recomendaciones de TV entre semana & $77(73.3 \%)^{* *}$ & $35(70.0 \%)$ & $42(76.4 \%)$ & $.542(1) / .072$ \\
\hline Cumplimiento de las recomendaciones de TV el finde semana & $36(34.3 \%)$ & $14(28.0 \%)$ & $22(40.0 \%)$ & $1.674(1) / .196$ \\
\hline Cumplimiento de las recomendaciones de videojuegos & $73(69.5 \%)$ & $27(54.0 \%)$ & $46(83.8 \%)$ & $10.857(1)^{* *} / .322$ \\
\hline Cumplimiento de las recomendaciones de videojuegos entre semana & $86(81.9 \%)^{* *}$ & $35(70 \%)$ & $51(92.7 \%)$ & $9.128(1)^{* *} / .295$ \\
\hline Cumplimiento de las recomendaciones de videojuegos el fin de semana & $69(65.7 \%)$ & $24(48 \%)$ & $45(81.8 \%)$ & $13.294(1)^{* *} / .356$ \\
\hline Cumplimiento de las recomendaciones de ordenador & $77(73.3 \%)$ & $35(70.0 \%)$ & $42(76.4 \%)$ & $.542(1) / .461$ \\
\hline Cumplimiento de las recomendaciones de ordenador entre semana & $86(81.9 \%)^{* *}$ & $40(80.0 \%)$ & $46(83.6 \%)$ & $.234(1) / .047$ \\
\hline Cumplimiento de las recomendaciones de ordenador el fin de semana & $77(77.3 \%)$ & $35(70.0 \%)$ & $42(76.4 \%)$ & $.542(1) / .072$ \\
\hline Cumplimiento de las recomendaciones de teléfono móvil & $60(57.1 \%)$ & $28(56.0 \%)$ & $32(58.2 \%)$ & $.051(1) / .022$ \\
\hline Cumplimiento de las recomendaciones de teléfono móvil entre semana & $83(79.0 \%)^{* *}$ & $36(72.0 \%)$ & $47(85.5 \%)$ & $1.863(1) / .165$ \\
\hline Cumplimiento de las recomendaciones de teléfono móvil el fin de semana & $58(55.2 \%)$ & $27(54.0 \%)$ & $31(56.4 \%)$ & $.059(1) / .024$ \\
\hline Cumplimiento de las recomendaciones de tiempo de pantalla & $0(0.0 \%)$ & $0(0.0 \%)$ & $0(0.0 \%)$ & $.449(1) /-.065$ \\
\hline $\begin{array}{l}\text { Cumplimiento de las recomendaciones de tiempo de pantalla entre } \\
\text { semana }\end{array}$ & $3(2.9 \%)$ & $2(4.0 \%)$ & $1(1.8 \%)$ & $.449(1) /-.065$ \\
\hline $\begin{array}{l}\text { Cumplimiento de las recomendaciones de tiempo de pantalla el fin } \\
\text { de semana }\end{array}$ & $0(0.0 \%)$ & $0(0.0 \%)$ & $0(0.0 \%)$ & \\
\hline
\end{tabular}

Nota: ${ }^{*}=p<0.05 ;{ }^{* *}=p<0.01$. Las asociaciones en función del género (hombre, mujer) aparecen reflejadas en la parte derecha de la tabla ( $x 2$ (gl) / V Cramer). Las diferencias en función del tipo de jornada (semana y fin de semana) se analizaron en la columna de muestra total ( $n=105)$ en cada uno de los medios tecnológicos de pantalla (TV, ordenador, videojuegos, móvil y totales). La significatividad se marca en el tipo de jornada de mayor valor.

\section{Discusión}

El aumento del tiempo que pasan los adolescentes frente a una pantalla se ha incrementado en los últimos ańos en los países desarrollados (Pate et al., 2011).En base a ello, resultan necesarios más estudios en la actualidad que analicen el tiempo de uso de estos dispositivos electrónicos. Por tanto, el objetivo del estudio fue tanto analizar el tiempo diario de uso sedentario de cuatro medios tecnológicos de pantalla (i.e., $\mathrm{TV}$, ordenador, videojuegos y teléfono móvil), como identificar el grado de cumplimiento de las recomendaciones de pantalla, tanto de manera independiente para cada medio tecnológico como en su conjunto.

Los resultados de este estudio señalan que los adolescentes pasan una media diaria de 6 horas y 47 minutos utilizando estos dispositivos electrónicos. Estos resultados son bastante superiores a otros estudios realizados con adolescentes españoles en los que se encontró que el tiempo medio en los medios tecnológicos evaluados en el presente estudio oscilaba entre un rango de 3 horas (Valencia et al., 2014)y 4 horas (Aibar et al., 2015) aproximadamente. Sin embargo, otros estudios internacionales han reportado,en la misma línea que el presente estudio, altos niveles de tiempo de pantalla. Por ejemplo, Leatherdale, y Ahmed (2011) realizaron un estudio en una muestra de 51.922 adolescentes canadienses en la que se registró un valor medio de casi 8 horas de tiempo de pantalla. La inclusión de diferentes medios tecnológicos y su rápida incorporación en la vida diaria de uso cotidiano puede explicar estas diferencias entre estudios relativamente próximos en el tiempo.

Con relación al cumplimiento total de las recomendaciones de tiempo de pantalla, ninguno de los adolescentes de esta muestra destina una media inferior a 2 horas diarias de tiempo de pantalla. Estos resultados son incluso más negativos que los reportados en el Health Behaviour in School-aged Childrenstudy; A WHO cross-National Survey, realizado en 39 países, en los que se encontró que un 77\% no cumplía las recomendaciones de tiempo de pantalla, situándose en un 68\% en España (Melkevik, Torsheim, Iannotti, \& Wold, 2010). Los resultados encontrados son tremendamente preocupantes ya que numerosas revisiones sistemáticas han evidenciado numerosas consecuencias negativas derivadas del uso elevado de tiempo de pantalla (e.g., baja condición física, baja autoestima, mala calidad del sueño; Carson et al., 2016). Teniendo en cuenta estos resultados y considerando la introducción en el mercado de consumo de nuevos aparatos electrónicos como los smartphones o las tablets, con un alto uso por parte de los adolescentes, se plantea la necesidad de replantear las recomendaciones establecidas sobre el uso delos diferentes medios tecnológicos de pantalla (Tremblay et al., 2011). 
Si analizamos independientemente los diferentes aparatos electrónicos,el tiempo de pantalla dedicado a ver la TV es el más utilizado y el que presenta un menor porcentaje de cumplimiento de las recomendaciones (37.1\%), seguido del teléfono móvil, los videojuegos y el ordenador. Los resultados encontrados en el tiempo diario dedicado a ver la TV están tanto en sintonía con estudios previos realizados en España(Abarca-Sos et al., 2016; Aibar et al., 2015; DevísDevís, Peiró-Velert, Beltrán-Carrillo, \& Tomás, 2009, 2012; Mielgo-Ayuso et al., 2017; Valencia et al., 2014) como con estudios internacionales (e.g., Arundell et al., 2016) en los que se observa una mayor prevalencia de uso de este dispositivo electrónico. Sin embargo, a diferencia de otros estudios realizados en España(Aibar et al., 2015; Peiró-Velert et al., 2012) el segundo medio tecnológico de pantalla más utilizado en este estudio fue el teléfono móvil. Este hecho puede deberse a que en la actualidad el 99\% de los adolescentes españoles posee teléfono móvil (Ditrendia, 2017). Las múltiples opciones y aplicaciones que disponen actualmente los smartphones (e.g., WhatsApp, redes sociales, reproductor de música, etc.), unido a las tarifas existentes con conexión permanente a internet, puede estar generando un aumento progresivo en el tiempo dedicado a la utilización de estos dispositivos (Gutiérrez, de Fonseca, \& Rubio, 2016).

Asimismo, el segundo objetivo del estudio fue analizar las posibles diferencias en el tiempo de pantalla en función del tipo de jornada y del género. En sintonía con previos estudios previos nacionales(Abarca-Sos et al., 2016; Devís-Devís et al., 2009, 2012; Martínez et al., 2012; Mielgo-Ayuso et al., 2017) e internacionales(Rey-López et al., 2010), los adolescentes hacen un mayor uso de las pantallas los fines de semana. Este hecho podría deberse a un mayor tiempo libre los fines de semana en el que no tienen la obligación de asistir a las 6 horas diarias lectivas. Con relación al género, los resultados encontrados están en línea con el estudio de Valencia et al. (2014) en el que los chicos presentaron valores significativamente superiores en el uso diario total de tiempo de pantalla. A pesar de que no se observaron diferencias significativas relativas a la TV, el ordenador y el teléfono móvil, las diferencias podrían estar explicadas por los valores significativamente superiores de los chicos en el uso de videojuegos, tal y como ocurre en otros estudios (Aibar et al., 2015; Noriega et al., 2015; Valencia et al., 2014). Un carácter más asociado al género masculino de los videojuegos (Paaßen, Morgenroth, \&Stratemeyer, 2017), y su futura consideración en el manual diagnóstico y estadístico de los trastornos mentales como trastorno oficial (i.e., Internet Gaming Disorder), sugiere la necesidad de prestar mayor atención hacia este comportamiento en programas de intervención educativa, especialmente en el género masculino (Irles, Gomis, Campos, \& González, 2017).

En base al incesante desarrollo tecnológico parece necesario reformular las recomendaciones de tiempo de pantalla en adolescentes, establecidas hace casi dos décadas por la American Association of Pediatrics (2001), adaptando la limitación de 2 horas de ocio a una cifra más apropiada al contexto tecnológico en el que nos encontramos y más específica a cada uno de los medios tecnológicos existentes.

Independientemente de las posibles modificaciones de las recomendaciones, y ante los alarmantes resultados encontrados, y atendiendo a algunas recomendaciones establecidas por diferentes estudios (e.g., Aap Council on Communications and Media, 2016; Altenburg, Kist-van Holthe, \& Chinapaw, 2016; Bartel, Scheeren, \& Gradisar, 2018; Friedrich, Polet, Schuch, \& Wagner, 2014; Schmidt, et al., 2012; Totland et al., 2013), sugerimos algunas recomendaciones que podrían ser tenidas en cuenta para reducir el tiempo de pantalla en adolescentes:

- Evitarla presencia de medios tecnológicos en el dormitorio, el instituto (salvo demanda del profesorado) y durante las comidas.

- Evitar el uso de pantalla de teléfonos móviles, tablets, ordenadores y/o consolas una hora antes de irse a dormir para no perjudicar el ciclo de sueño.

- Evitar que los niños y adolescentes hagan sus tareas escolares mientras ven la televisión y/o utilizan cualquier dispositivo electrónico de manera simultánea.

- Evitar utilizar dispositivos electrónicos para distraer o calmar la ansiedad.

- Evitar que el tiempo de pantalla no reemplace el tiempo requerido para dormir, hacer actividad física u otras conductas relacionadas con la salud.

- Establecer un horario y un tiempo máximo de uso recreativo de los dispositivos electrónicos.

- Bloquear algunas aplicaciones y restringir el uso de determinadas páginas web con un contenido poco educativo.

- Actuar con ejemplo como figura paternal o maternal en el uso limitado de medios tecnológicos en su tiempo de ocio.

- Promover un tiempo de ocio activo, y la actividad física y deportiva como alternativa al tiempo sedentario de pantalla.

\section{Conclusiones}

Los adolescentes de este estudio dedican un tiempo excesivo a la utilización de los diferentes medios tecnológicos de pantalla. Entre los resultados cabe destacar que ninguno de los adolescentes cumple las recomendaciones diarias de tiempo de pantalla. Entre semana solo un 3\% de la muestra cumple dichas recomendaciones. El tipo de jornada y el género condicionan el tiempo medio diario de uso estos dispositivos electrónicos, siendo superior los fines de semana y por parte de los chicos. En base a los resultados encontrados, parece 
conveniente reconsiderar las recomendaciones de tiempo de pantalla decada uno de los dispositivos electrónicos y del total de tiempo de pantalla debido a que los valores quedan enormemente alejados a la realidad tecnológica actual. El diseño de estrategias específicas para limitar el tiempo de uso de los diferentes medios tecnológicos de pantalla se configura como uno de los objetivos prioritarios dados los alarmantes niveles de ocio sedentario. Para ello, resulta necesario hacer especial énfasis en la restricción del tiempo de TV, teléfono móvil, y de uso de videojuegos específicamente en chicos.

\section{Financiación}

Este trabajo se encuentra financiado por el Ministerio de Economía y Competitividad (MINECO; EDU2013-42048-R), el Gobierno de Aragón y el Fondo Social Europeo.

\section{Referencias bibliográficas}

1. AAP Councilon Communications and Media (2016). Media use in school-aged children and adolescents. Pediatrics, 138(5), e20162592.

2. Abarca-Sos, A., Bois, J. E., Aibar, A., Julián, J. A., Generelo, E., \& Zaragoza, J. (2016). Sedentary behaviors by type of day and physical activity in spanish adolescents: a socio-ecological approach. Perceptual and Motor Skills, 122(1), 286-298.

3. Altenburg, T. M., Kist-van Holthe, J., \& Chinapaw, M. J. (2016). Effectiveness of intervention strategies exclusively targeting reductions in children's sedentary time: a systematic review of the literature. International Journal of Behavioral Nutrition and Physical Activity, 13(1), 65 doi:10.1186/s12966-016-0387-5

4. Aibar, A., Bois, J. E., Zaragoza, J., Bru, N., Paillard, T., \&Generelo, E. (2015). Adolescents' sedentary behaviors in two European cities. Re search Quarterly for exercise and Sport, 86(3), 233-243. doi:10.1080/027 01367.2015.1039891

5. American Association of Pediatrics (2001). Children, adolescents, and television. Pediatrics, 107(2), 423-426.doi:10.1542/peds.107.2.423

6. Arundell, L., Fletcher, E., Salmon, J., Veitch, J., \& Hinkley, T. (2016). A systematic review of the prevalence of sedentary behavior during the after-school period among children aged 5-18 years. International Journal of Behavioral Nutrition and Physical Activity, 13(1), 93. doi:10.1186/ s12966-016-0419-1

7. Bartel, K., Scheeren, R., \& Gradisar, M. (2018). Altering adolescents' pre-bedtime phone use to achieve better sleep health. Health Communi cation, 1-7. doi:10.1080/10410236.2017.1422099

8. Carson, V., Hunter, S., Kuzik, N., Gray, C. E., Poitras, V. J., Chaput, J. P., ... \& Kho, M. E. (2016). Systematic review of sedentary behaviour and health indicators in school-aged children and youth: an update. Applied Physiology, Nutrition, and Metabolism, 41(6), S240S265. doi:10.1186/1479-5868-8-98.

9. Costigan, S. A., Barnett, L., Plotnikoff, R. C., \& Lubans, D. R. (2013). The health indicators associated with screen-based sedentary behavior among adolescent girls: A systematic review. Journal of Adolescent Health, 52(4), 382-392. doi:10.1016/j.jadohealth.2012.07.018

10. Devís-Devís, J., Peiró-Velert, C., Beltrán-Carrillo, V. J., \& Tomás, J. M. (2009). Screen media time usage of 12-16 year-old Spanish school adolescents: effects of personal and socioeconomic factors, season and type of day. Journal of Adolescence, 32(2), 213-231. doi:10.1016/j.adolescence.2008.04.004

11. Devís-Devís, J., Peiró-Velert, C., Beltrán-Carrillo, V. J., \& Tomás, J. M. (2012). Brief report: Association between socio-demographic factors, screen media usage and physical activity by type of day in Spanish adolescents. Journal of Adolescence, 35(1), 213-218. doi:10.1016/j.adolescence.2010.11.009

12. Digital Marketing Trends (Ditrendia, 2017). Informe Mobile en España y en el Mundo 2016. Recuperado de: http://www.amic.media/media/ files/file_352_1289.pdf

13. Friedrich, R. R., Polet, J. P., Schuch, I., \& Wagner, M. B. (2014). Effect of intervention programs in schools to reduce screen time: a meta-analysis. Jornal de Pediatria, 90(2), 232-241. doi:10.1016/j. jped.2014.01.003

14. Gómez, D. M., Veiga, O. L., Zapatera, B., Cabanas-Sánchez, V., Gomez-Martinez, S., Martinez-Hernández, D., \& Marcos, A. (2012). Patterns of sedentary behavior and compliance with public health recommendations in Spanish adolescents: the AFINOS study Patrones de sedentarismo y cumplimiento de las recomendaciones de salud pública en adolescentes. Cadernos de Saúde Pública, 28(12), 2237-2244.

15. Gutiérrez, J. D. S., de Fonseca, F. R., \& Rubio, G. (2016). Cell-phone addiction: a review. Frontiers in Psychiatry, 7, 175. doi:10.3389/fpsyt.2016.00175

16. Inchley, J. C., Currie, D. B., Young, T., Samdal, O., Torsheim, T., Augustson, L., ... \& Barnekow, V. (Eds.) (2016). Growing up unequal: gender and socioeconomic differences in young people's health and well-being: Health Behaviour in School-aged Children (HBSC) study: international report from the 2013/2014 survey. (Health Policy for Children and Adolescents; No. 7). Denmark: WHO Regional Office for Europe.

17. Irles, D. L., Gomis, R. M., Campos, J. C. M., \& González, S. T. (2017). Validación española de la Escala de Adicción a Videojuegos para Adolescentes (GASA). Atención Primaria. doi:10.1016/j.aprim.2017.03.015

18. Leatherdale, S.T., \& Ahmed, R. (2011). Screen-based sedentary behaviours among a nationally representative sample of youth: are Canadian kids couch potatoes? Chronic Diseases and Injuries in Canada, 31(4), 141-146.

19. Lissak, G. (2018). Adverse physiological and psychological effects of screen time on children and adolescents: Literature review and case study. Environmental Research, 164, 149-157. doi:10.1016/j.envres.2018.01.015

20. Melkevik, O., Torsheim, T., Iannotti, R. J., \& Wold, B. (2010). Is spending time in screen-based sedentary behaviors associated with less physical activity: a cross national investigation. International Journal of Behavioral Nutrition and Physical Activity, 7(1), 46. doi: 10.1186/14795868-7-46

21. Mielgo-Ayuso, J., Aparicio-Ugarriza, R., Castillo, A., Ruiz, E., Avila, J. M., Aranceta-Bartrina, J., ... \& González-Gross, M. (2017). Sedentary behavior among Spanish children and adolescents: findings from the ANIBES study. BMC Public Health, 17(1), 94. doi:10.1186/s12889-0174026-0

22. Norris, E., Hamer, M., \& Stamatakis, E. (2016). Active video games in schools and effects on physical activity and health: a systematic review. The Journal of Pediatrics, 172, 40-46. doi:10.1016/j. jpeds.2016.02.001

23. Paaßen, B., Morgenroth, T., \& Stratemeyer, M. (2017). What is a true gamer? The male gamer stereotype and the marginalization of women in video game culture. Sex Roles, 76(7-8), 421-435. doi:10.1007/s11199. 016-0678-y

24. Pate, R. R., Mitchell, J. A., Byun, W., \& Dowda, M. (2011). Sedentary behaviour in youth. British Journal of Sports Medicine, 45(11), 906-913. doi:10.1136/bjsports-2011-090192 
25. Pearson, N., Haycraft, E., Johnston, J. P., \& Atkin, A. J. (2017). Sedentary behaviour across the primary-secondary school transition: a systematic review. Preventive Medicine, 94, 40-47. doi:10.1016/j.ypmed.2016.11.010

26. Rey-López, J. P., Ruiz, J. R., Ortega, F. B., Verloigne, M., VicenteRodriguez, G., Gracia-Marco, L.,... \& Moreno, L. (2011). Reliability and validity of a screen time-based sedentary behaviour questionnaire for adolescents: the HELENA study. The European Journal of Public Health, 22(3), 373-377. doi:10.1093/eurpub/ckr040.

27. Rey-López, J. P., Vicente-Rodriguez, G., Ortega, F. B., Ruiz, J. R., Martinez-Gómez, D., De Henauw, S., ... \& Castillo, M. J. (2010). Sedentary patterns and media availability in European adolescents: The HELENA study. Preventive Medicine, 51(1), 50-55. doi:10.1016/j.ypmed.2010.03.013

28. Schmidt, M. E., Haines, J., O’brien, A., McDonald, J., Price, S., Sherry, B., \& Taveras, E. M. (2012). Systematic review of effective strategies for reducing screen time among young children. Obesity, 20(7), 1338-1354. doi:10.1038/oby.2011.348

29. Totland, T. H., Bjelland, M., Lien, N., Bergh, I. H., Gebremariam, M. K., Grydeland, M., ... \& Andersen, L. F. (2013). Adolescents' prospective screen time by gender and parental education, the mediation of parental influences. International Journal of Behavioral Nutrition and Physical Activity, 10(1), 89. doi:10.1186/1479-5868-10-89

30. Tremblay, M. S., Aubert, S., Barnes, J. D., Saunders, T. J., Carson, V., Latimer-Cheung, A. E., ... \& Chinapaw, M. J. (2017). Sedentary Behavior Research Network (SBRN) - Terminology consensus project process and outcome. International Journal of Behavioral Nutrition and Physical Activity, 14(1), 75.doi: 10.1186/s12966-017-0525-8

31. Tremblay, M. S., Carson, V., Chaput, J. P., Connor Gorber, S., Dinh, T., Duggan, M., ... \& Zehr, L. (2016). Canadian 24-hour movement guidelines for children and youth: An Integration of physical activity, sedentary behaviour, and sleep. Applied Physiology, Nutrition, and Metabolism, 41(6), S311-S327. doi:10.1139/apnm-2016-0151.

32. Tremblay, M. S., LeBlanc, A. G., Kho, M. E., Saunders, T. J., Larouche, R., Colley, R. C., Goldfield, G., \& Gorber, S. C. (2011). Systematic review of sedentary behaviour and health indicators in school-aged children and youth. International Journal of Behavioral Nutrition and Physical Activity, 8(1), 98-101. doi:10.1186/1479-5868-8-98

33. Twenge, J. M., Krizan, Z., \& Hisler, G. (2017). Decreases in self-reported sleep duration among US adolescents 2009-2015 and association with new media screen time. Sleep Medicine, 39, 47-53. doi:10.1016/j. sleep.2017.08.013

34. Valencia-Peris, A., Devís-Devís, J., \& Peiró-Velert, C. (2014). El uso sedentario de medios tecnológicos de pantalla: perfil sociodemográfico de los adolescentes españoles. Retos. Nuevas tendencias en Educación Física Deporte y Recreación, 26, 21-26.

35. Verloigne, M., Loyen, A., Van Hecke, L., Lakerveld, J., Hendriksen, I., De Bourdheaudhuij, I., ... \& van der Ploeg, H. P. (2016). Variation in population levels of sedentary time in European children and adolescents according to cross-European studies: a systematic literature review within DEDIPAC. International Journal of Behavioral Nutrition and Physical Activity, 13(1), 69. doi: 10.1186/s12966-016-0395-5 